\title{
LEARN: Localized Energy Aware Restricted Neighborhood Routing for Ad Hoc Networks
}

\author{
Yu Wang ${ }^{\dagger}$, Wen-Zhan Song ${ }^{\ddagger}$, Weizhao Wang*, Xiang-Yang Li* $^{*}$, Teresa A. Dahlberg ${ }^{\dagger}$ \\ ${ }^{\dagger}$ Department of Computer Science, University of North Carolina at Charlotte, Charlotte, NC, USA \\ ${ }^{\ddagger}$ School of Engineering and Computer Science, Washington State University, Vancouver, WA, USA \\ ${ }^{*}$ Department of Computer Science, Illinois Institute of Technology, Chicago, IL, USA
}

\begin{abstract}
In this paper, we address the problem of energy efficient localized routing in wireless ad hoc networks. Numerous energy aware routing protocols were proposed to seek the power efficiency of routes. Among them, several geographical localized routing protocols were proposed to help making smarter routing decision using only local information and reduce the routing overhead. However, most of the proposed localized routing methods cannot theoretically guarantee the power efficiency of their routes. In this paper, we give the first localized routing algorithm, called Localized Energy Aware Restricted Neighborhood routing (LEARN), which can guarantee the power efficiency of its route asymptotically almost sure. Given destination node $t$, an intermediate node $u$ will only select a certain neighbor $v$ such that $\angle v u t \leq \alpha$ for a parameter $\alpha<\frac{\pi}{3}$ in our LEARN method. We theoretically prove that for a network, formed by nodes that are produced by a Poisson distribution with rate $n$ over a compact and convex region $\Omega$ with unit area, when the transmission range $r_{n}=\sqrt{\frac{\beta \ln n}{\pi n}}$ for some $\beta>\frac{\pi}{\alpha}$, our LEARN routing protocol will find the route for any pair of nodes asymptotically almost sure. When the transmission range $r_{n}=\sqrt{\frac{\beta \ln n}{\pi n}}$ for some $\beta<\frac{\pi}{\alpha}$, the LEARN routing protocol will not be able to find the route for any pair of nodes asymptotically almost sure. We also conducted simulations to study the performance of LEARN and compare it with a typical localized routing protocol (GPSR) and a global ad hoc routing protocol (DSR).
\end{abstract}

\section{INTRODUCTION}

Wireless ad hoc network (including sensor network) draws lots of attentions in recent years due to its potential applications in various areas and it intrinsically has many special characteristics and some unavoidable limitations, compared with traditional fixed infrastructure networks. Energy conservation and scalability are probably two most critical issues in designing protocols for wireless ad hoc networks, because wireless devices are usually powered by batteries only and have limited computing capability while the number of such devices could be very large. In this paper we focus on designing routing protocols for wireless ad hoc networks which can achieve both energy efficiency by carefully selecting the forwarding neighbors and high scalability by using only local information to make routing decisions.

A number of energy efficient routing protocols [1]-[8] have been proposed recently using various techniques (dynamic transmission power adjustment, adaptive sleeping, topology control, multi-path routing, directional antennas, etc). Most of the proposed energy-aware routing methods take into account the energy-related metrics instead of traditional routing metrics such as delay or hop count. To select the optimal energy route, those methods usually need the globe information of the whole network, and each node need also maintaining the routing table as protocol states. In opposition to the energy efficient routing protocols, several stateless routing protocols, particularly, many localized geographic routing protocols [8][12] have been proposed to improve the scalability. In those localized routing protocols, by assuming each node have position information, the routing decision is made at each node by using only local neighborhood information. They do not need the dissemination of route discovery information, and no routing tables are maintained at each node. Previous localized routing protocols are not energy efficient, i.e., the total power consumed by their route could be very large in the worst case [12], [14]. Recently, some energy-aware localized routing protocols [6], [8], [13] take the energy concern during the routing decision. However, all of them cannot theoretically guarantee the power efficiency of their routes.

We study energy-efficient localized routing protocol for ad hoc networks. Our main contributions are follows.

- New Localized Routing Protocol: We propose a new localized routing protocol, called localized energy aware restricted neighborhood routing (LEARN). In LEARN, whenever possible, the node selects the neighbor inside a restricted neighborhood (defined by a parameter $\alpha$ ) that has the largest energy mileage (i.e., the distance traveled per unit energy consumed) as the next hop node. If no such neighbor inside the restricted neighborhood, it acts as greedy routing. The guarantee of delivery can be achieved by using face routing as the backup.

- Power Efficiency of LEARN: We theoretically prove that LEARN is power efficient, i.e., when LEARN routing finds a path from the source node to the target node, the total energy consumption of the found path is within a constant factor of the optimum. Notice that, LEARN routing is the first localized routing which can theoretically guarantee the power efficiency of its routes. In addition, we also prove that the total Euclidean length of the found path is within a constant factor of the optimum.

- Critical Transmission Range for LEARN: We theoretically prove that for a network, formed by nodes that are produced by a Poisson distribution with rate $n$ over a compact and convex region $\Omega$ with unit area, 
when the transmission range $r_{n}=\sqrt{\frac{\beta \ln n}{\pi n}}$ for some $\beta>\frac{\pi}{\alpha}$, our LEARN routing protocol will find the route for any pair of nodes asymptotically almost sure. When the transmission range $r_{n}=\sqrt{\frac{\beta \ln n}{\pi n}}$ for some $\beta<\frac{\pi}{\alpha}$, the LEARN routing protocol will not be able to find the route for any pair of nodes asymptotically almost sure.

- Simulation for LEARN: We conducted extensive simulations to study the performance of LEARN and compare it with a typical localized routing protocol (GPSR) and a global ad hoc routing protocol (DSR). The simulation results show that our LEARN routing protocol has good performances in random networks.

The rest of the paper is organized as follows. We first introduce the network model and review related works in Section II. Then we present our localized energy aware routing protocol (LEARN) and prove its energy efficiency in Section III. In Section IV, we study the asymptotic transmission range for our LEARN routing. Some implementation issues and possible improvements are discussed in Section V. We compare the performance of our method with existing routing methods in Section VI and conclude our paper in Section VII.

\section{Preliminaries AND Network MOdel}

\section{A. Network Model}

There is a set $V$ of $n=|V|$ of wireless devices (called nodes hereafter) distributed in a region $\Omega$. Typically, we assume that the region $\Omega$ is a unit-area square or a unit area disk. Each node is assigned a unique ID $i \in[1, n]$. Additionally, each node knows its position information either through a low-power GPS receiver or some other ways. By one-hop broadcasting, each node $i$ can gather the location information of all nodes within its transmission range. We assume that every wireless node has a uniform transmission range $r$. The multi-hop wireless network is then modeled by a communication graph $G=(V, E)$, where $E$ is the set of $m=|E|$ undirected links and a link $(u, v)$ belongs to $E$ if and only if the Euclidean distance between $u$ and $v$ is at most $r$. Hereafter, we use $\|u-v\|$ to denote the Euclidean distance between node $u$ and node $v$. For a undirected link $u v \in G$, we use $\|u v\|$ to denote its length. We also assume that each node $i$ can dynamically adjust its transmission power based on the neighboring node it wants to communicate. We further assume that the energy needed to support the transmission of a unit amount of data over a link $u v$ is $\mathbf{c}(\|u v\|)$, where $\mathbf{c}(x)$ is a non-decreasing function on $x$. Let $\mathbf{r}_{0}$ be the value such that $\frac{\mathbf{r}_{0}}{\mathbf{c}\left(\mathbf{r}_{0}\right)}=\max _{x} \frac{x}{\mathbf{c}(x)}$. We call $\frac{\mathbf{r}_{0}}{\mathbf{c}\left(\mathbf{r}_{0}\right)}$ as the maximum energy mileage ${ }^{1}$ under energy consumption model $\mathbf{c}(x)$. We assume that the energy mileage $\frac{x}{\mathbf{c}(x)}$ is an increasing function when $x<\mathbf{r}_{0}$ and is a decreasing function when $x>\mathbf{r}_{0}$. For example, in the literature it is commonly assumed that $\mathbf{c}(\|u v\|)=E_{0}\left(\|u v\|^{\gamma}+c\right)$, where $E_{0}, \gamma \geq 2$, and $c$ are constants depending on the transmission environment and device. For the sake of the analysis, it is often

\footnotetext{
${ }^{1}$ Here we assume that the derivative of function $d\left(\frac{\mathbf{c}(x)}{x}\right) / d x=$ $\frac{\mathbf{c}^{\prime}(x) x-\mathbf{c}(x)}{x^{2}}$ is monotone increasing, thus, $\mathbf{r}_{0}$ is unique.
}

assumed that $E_{0}=1$ and $\gamma=2$, i.e., $\mathbf{c}(\|u v\|)=\|u v\|^{2}+c$. Under this specific energy consumption model, we can show that the maximum energy mileage is $\mathbf{r}_{0}=\sqrt{c}$. Notice that when $\mathbf{r}_{0}>r$, the best length of the forwarding link is $r$.

\section{B. Problem Specification}

A routing protocol is said to be a localized protocol if, given the information of the source node $s$ and the target node $\mathbf{t}$ and the $k$-hop neighborhood information, the current node $u$ can decide which neighboring node $v$ to forward the message. Here $k$ is a constant, usually 1 or 2 . Given a routing method $\mathcal{A}$, let $\mathbf{P}_{\mathcal{A}}(s, \mathbf{t})$ be the path found by $\mathcal{A}$ to connect the source node $s$ and the target node $\mathbf{t}$. A routing method $\mathcal{A}$ is called power efficient if for every pair of nodes $s$ and $\mathbf{t}$, the energy consumption of path $\mathbf{P}_{\mathcal{A}}(s, \mathbf{t})$ is within a constant factor of the least energy-consumption path connecting $s$ and $\mathbf{t}$ in the network. In this paper, we want to design a localized power efficient routing method $\mathcal{A}$.

For a general network, it was shown in [14] that there is no deterministic localized routing method that is power efficient. Kuhn et al. [14] constructed an example to show that any deterministic (randomized) geometric ad-hoc routing algorithm has (expected) cost (either hop-count, Euclidean distance, or energy-consumption) $\Omega\left(O P T^{2}\right)$, where $O P T$ is the optimum cost. We then concentrate on designing a localized routing method that is power efficient with high probability. Here a routing method is power efficient with high probability if (1) with high probability, the routing method will find a path successfully; and (2) with high probability, the found path is power efficient.

\section{Related Work on Localized Routing}

The geometric nature of the multi-hop ad-hoc networks allows the promising idea: localized routing protocols. The most popular localized routing is greedy routing where the current node $u$ always finds the next relay node $v$ such that the distance $\|\mathbf{t}-v\|$ is the smallest among all neighbors of $u$. Here $\mathbf{t}$ is the destination. There are several variations of greedy routing which use various criteria of "greedy" instead of nearest to the destination. For example, compass routing [15] finds the next relay node $v$ such that the angle $\angle v u$ is the smallest; farthest neighbor routing finds the farthest node as the forwarding node; most forwarding routing [8] finds the relay node $v$ such that $\left\|\mathbf{t}-v^{\prime}\right\|$ is the smallest, where $v^{\prime}$ is the projection of $v$ on segment $u \mathbf{t}$. Though greedy routing and its variations were widely used for wireless networks, it is easy to construct an example to show that greedy algorithm will not succeed to reach the destination but fall into a local minimum (a node without any "better" neighbors).

To guarantee the packet delivery, face routing is proposed by [15]. The idea of face routing is to walk along the faces which are intersected by the line segment between the source and the destination. In each face, it uses the right hand rule to explore the boundaries. [14], [15] proved that face routing guarantees to reach the destination after traversing at most $O(n)$ edges when the network topology is a planar graph. Though face 
routing terminates in linear time, it is not satisfactory, since already a simple flooding algorithm can terminate in $O(n)$ steps. Then Kuhn et al. [11], [14] proposed two refined face routing methods, in which, restricted search areas are used to avoid exploring the complete boundary of faces.

Greedy routing is a simple and efficient method but cannot guarantee the packet delivery, while face routing can guarantee the delivery but may take a very long exploration $(O(n)$ steps). One natural approach to improve the performance of localized routing is to combine greedy routing and face routing by using face routing to recover the routing after simple greedy method fails in local minimum. Many wireless protocols used this approach [8]-[12].

Although face routing and greedy face routing can guarantee the packet delivery when underlying topology is planar and some of localized routing protocols guarantee the delivery if some special geometry structures are used as the routing topology, none of these guarantees the ratio of the distance traveled by the packets over the minimum possible. Bose and Morrin [16] proposed a method to bound this ratio using the Delaunay triangulation. They showed that the distance traveled by the packet is within a constant factor of the distance between the source and the destination. However, recently, Wang and Li [17] showed the Delaunay based routing [16] is not power efficient (i.e., the power spent by the route could be sufficiently larger than the optimal least-power path).

\section{Related Work on Power Efficient Routing}

Since energy is a scarce resource which limits the life of the network, a number of energy efficient routing protocols [1][8] have been proposed recently using a variety techniques. Classical routing algorithm may be adapted to take into account energy-related criteria rather than classical metrics such as delay or hop distance. Most of the proposed energyaware metrics are defined as a function of the energy required to communicate on a link [1], [2], [5] or a function of the nodes remaining lifetime [3]. However, to minimize the global consumed energy of selected route, most of minimum energy routing algorithms are centralized algorithms as [3]-[5]. In this paper, we focus on stateless localized routing. Thus, we only review the following related work about power efficient techniques for "local" routing which address how to save energy when making routing decision.

In [13], Roosta proposed a localized routing method called probabilistic geographic routing to address power-aware routing for ad hoc and sensor networks. It selects the next hop probabilistically among a set of neighbor candidates and the neighbors assign the probability proportional to their residual energy and the link reliability. In [6], Melodia et al. proposed a partial topology knowledge forwarding for sensor network, where each node selects the shortest energy-weighted path based on local knowledge of topology. They assumed the neighborhood discovery protocol provides each node the local knowledge of topology within certain range. They gave a linear programming formulation to select the range which minimizes the energy expenditure of the network. Since the solution of the linear programming problem is not feasible in practice, they also proposed a distributed protocol to adjust the topology knowledge range. In [8], Stojmenovic and Lin proposed a power-aware localized routing which combining the cost metric based on remaining battery power at nodes and the power metric based on the transmission power related to distance between nodes. They proved the loop-free properties of their methods and show their efficiency by experiments. Stojmenovic and Datta [7] further combined the above method with face routing to guarantee the delivery. However, they provide no theoretical guarantee of power efficiency for all of their methods.

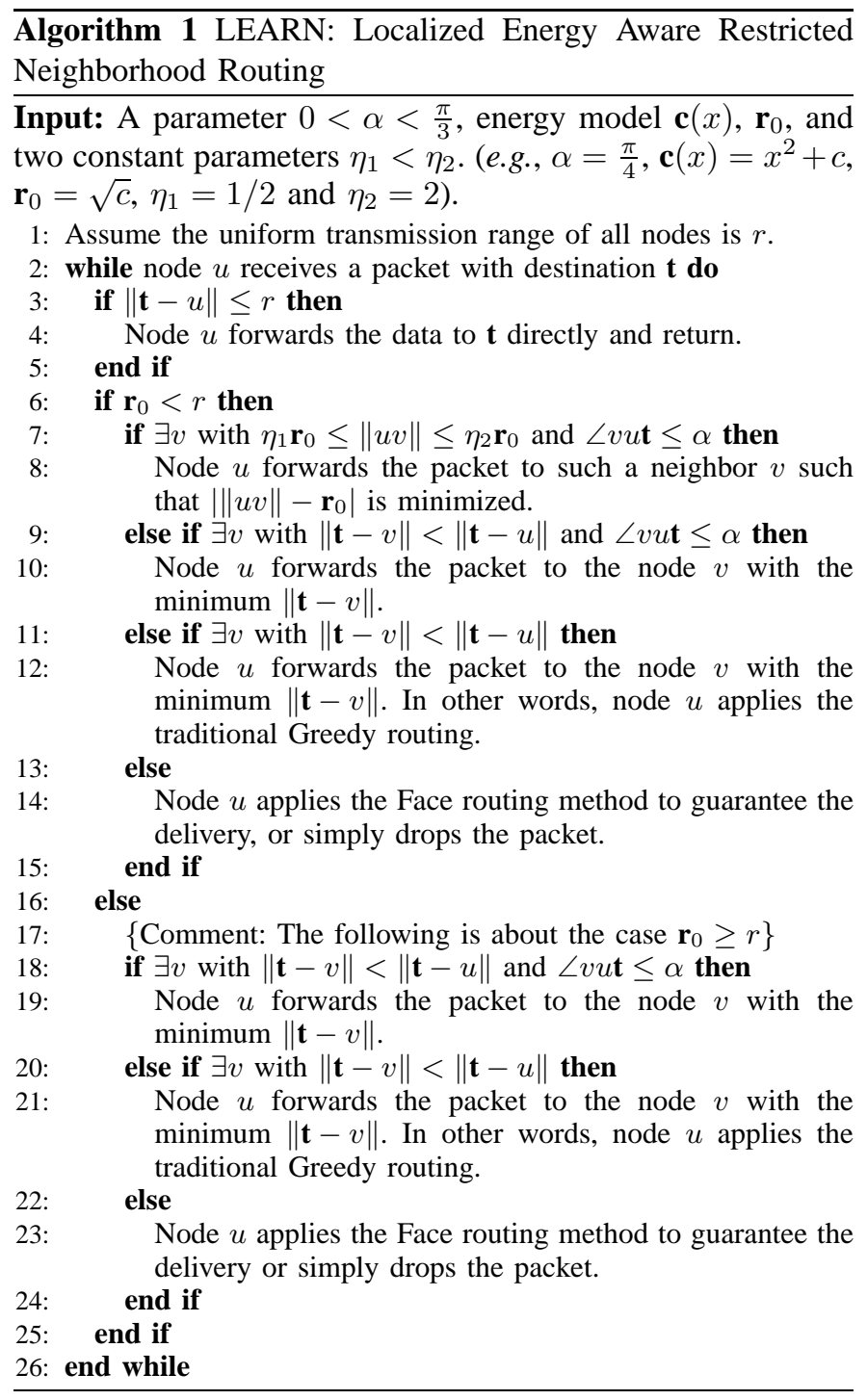

\section{Power EfFicient Localized Routing}

In this section, we describe in detail our power efficient localized routing method, called localized energy aware restricted neighborhood routing (LEARN). The definition of energy mileage provides us the insight in designing energy efficient routing (without considering the retransmissions here). 
Whenever possible, we should use link that has larger energy mileage. Additionally, to save the energy consumption, the total distance traveled should be as small as possible. Thus, intuitively, our localized energy aware restricted neighborhood routing protocol will work as follows. The current intermediate node $u$ with message will first find the "best" neighbor $v$ among all neighbors $w$ such that $\angle w u \mathbf{t} \leq \alpha$ for a parameter $\alpha<\pi / 3$. Here we define the "best neighbor" as the node $v$ such that $\frac{\|u v\|}{\mathbf{c}(\|u v\|)}$ is maximum among all such neighbors; the use of the angle $\alpha$ is to bound the total distance of the routing path. Algorithm 1 illustrates our localized energy aware routing protocol. Recall that $\mathbf{r}_{0}$ is the best link length that achieves that maximum energy mileage.

To make the later analysis easier, we call the routing algorithm LEARN if no Greedy routing and no Face routing is used when no node $v$ satisfying that $\angle v u \mathbf{t} \leq \alpha$. If greedy routing is applied afterward, then the routing protocol is called LEARN-G. Furthermore, if the Face routing is used at the end to get out of the local minimum, the routing protocol is called LEARN-GF. Figure 1 illustrates our localized routing algorithm. If no such neighbor $v$ exists, then we use the traditional greedy routing as shown in Figure 1(b).

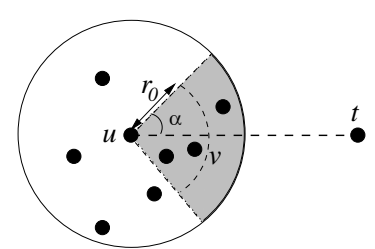

(a) forwarding inside $2 \alpha$ region

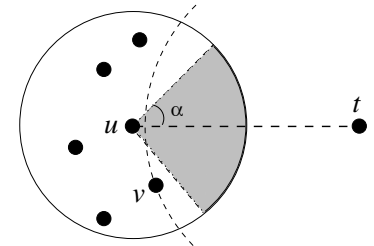

(b) greedy forwarding
Fig. 1. Illustrations of LEARN: (a) power efficient forwarding in $2 \alpha$-sector region, (b) traditional greedy forwarding when the sector region is empty.

Theorem 1: When LEARN routing indeed finds a path $\mathbf{P}_{\text {LEARN }}(s, \mathbf{t})$ from the source $s$ to the target $\mathbf{t}$, the total Euclidean length of the found path is within a constant factor $\|\mathbf{t}-s\|$, thus, a constant factor of the optimum.

Proof: We will prove that the total Euclidean length of $\mathbf{P}_{L E A R N}(s, \mathbf{t})$ is at most $\delta\|\mathbf{t}-s\|$ for any source $s$ and destination $\mathbf{t}$ for a constant $\delta=\frac{1}{1-2 \sin \frac{\alpha}{2}}$. Notice that $\alpha<$ $\pi / 3$. We prove it by induction on the number of hops. It is clearly true when the path has only one-hop. Assume that it is true for the path with $(k-1)$-hops. Then consider any path $v_{0} v_{1} v_{2} \cdots v_{k-1} v_{k}$ with $k$-hops. By induction, the length of path $v_{1} v_{2} \cdots v_{k-1} v_{k}$ is at most $\delta\left\|v_{k}-v_{1}\right\|$. Then it is sufficient to show that $\left\|v_{0} v_{1}\right\|+\delta\left\|v_{k}-v_{1}\right\| \leq \delta\left\|v_{k}-v_{0}\right\|$.

Let $\angle v_{0} v_{k} v_{1}=2 x$ and $\angle v_{1} v_{0} v_{k}=\theta \leq \alpha$. Then a simple geometry computation shows that $\frac{\left\|v_{0} v_{1}\right\|}{\left\|v_{k}-v_{0}\right\|-\left\|v_{k}-v_{1}\right\|}=$ $\frac{\sin (x+\pi / 2)}{\sin \left(\frac{\pi}{2}-x-\theta\right)}=\frac{\cos x}{\cos (x+\theta)}$. Notice that by our routing protocol, we have $v_{0} v_{k}$ is the longest link in triangle $v_{0} v_{1} v_{k}$. Thus, it is easy to show that we need $x<\frac{\pi}{2}-\theta$ and $x<\frac{\pi-\theta}{4}$. Simple computation shows that $\frac{\cos x}{\cos (x+\theta)} \leq \frac{1}{1-2 \sin \frac{\theta}{2}} \leq \frac{1}{1-2 \sin \frac{\alpha}{2}}$ for $x<\min \left(\frac{\pi}{2}-\theta, \frac{\pi-\theta}{4}\right)$. Thus, $\left\|v_{0} v_{1}\right\|+\delta\left\|v_{k}-v_{1}\right\| \leq$ $\delta\left\|v_{k}-v_{0}\right\|$. This finishes the proof.

We then show that our LEARN routing protocol is indeed energy efficient when it finds the path successfully. We prove it by two separate cases: $\mathbf{r}_{0} \geq r$ or $\mathbf{r}_{0}<r$.

Theorem 2: When LEARN routing indeed finds a path $\mathbf{P}_{L E A R N}(s, \mathbf{t})$ from the source $s$ to the target $\mathbf{t}$ and $\mathbf{r}_{0} \geq r$, the total energy consumption of the found path is within a constant factor of the optimum.

Proof: When $\mathbf{r}_{0} \geq r$, for any intermediate node $u$ with packets to forward to destination $\mathbf{t}$, our LEARN routing protocol will select the neighbor $v$ with $\angle v u \mathbf{t} \leq \alpha$ and $\|\mathbf{t}-v\|$ is minimized. See Figure 2 (a) for illustration.

Assume that the path $v_{0} v_{1} v_{2} \cdots v_{k-1} v_{k}$ is found by our routing protocol to connect source node $s=v_{0}$ and destination node $\mathbf{t}=v_{k}$. We first prove that for every two consecutive links $v_{i-1} v_{i}$ and $v_{i} v_{i+1},\left\|v_{i-1} v_{i}\right\|+\left\|v_{i} v_{i+1}\right\|>r$. Assume that this is not true, i.e., there are 3 consecutive nodes, say $u$, $v$, and $w$ on the path with $\|u v\|+\|v w\| \leq r$. Obviously, $w$ is neighbor of $u$ since $\|u w\| \leq\|u v\|+\|v w\| \leq r$. We can prove that $\angle w u \mathbf{t} \leq \alpha$ (the proof is omitted here due to space limit). Additionally, by the routing protocol we know that $\|\mathbf{t}-w\|<$ $\|\mathbf{t}-v\|$ and $\|\mathbf{t}-v\|<\|\mathbf{t}-u\|$. These contradict the selection of node $v$ by node $u$ for forwarding: it should select the node that is closest to destination $\mathbf{t}$ and clearly node $v$ is not (since neighbor $w$ is closer to than neighbor $v$ ).

Notice that since $\mathbf{r}_{0} \geq r$, then for any path connecting $v_{0}$ and $v_{k}$, its energy consumption is at least $\frac{\left\|v_{0}-v_{k}\right\|}{r} \mathbf{c}(r)$. This can be proved as follows. Let $x_{i}, 1 \leq i \leq k$ be the $i$ th link length of the path. Then its total energy consumption is $\sum_{i=1}^{k} \mathbf{c}\left(x_{i}\right)=\sum_{i=1}^{k} \frac{\mathbf{c}\left(x_{i}\right)}{x_{i}} \cdot x_{i} \geq \sum_{i=1}^{k} \frac{\mathbf{c}(r)}{r} \cdot x_{i}=$ $\frac{\mathbf{c}(r)}{r} \sum_{i=1}^{k} x_{i} \geq \frac{\left\|v_{0}-v_{k}\right\|}{r} \mathbf{c}(r)$.

Let $x_{i}=\left\|v_{i-1} v_{i}\right\|$. Theorem 1 implies that $\sum_{i=1}^{k} x_{i} \leq$ $\frac{1}{1-2 \sin \frac{\alpha}{2}}\left\|v_{0}-v_{k}\right\|$. Since $x_{i}+x_{i+1}>r$ for any $1 \leq i<k$, we have $k \leq 2\left\lceil\frac{\frac{\left\|v_{0}-v_{k}\right\|}{1-2 \sin \frac{\alpha}{2}}}{r}\right\rceil \leq 2\left\lceil\frac{1}{1-2 \sin \frac{\alpha}{2}}\right\rceil \cdot\left\lceil\frac{\left\|v_{0}-v_{k}\right\|}{r}\right\rceil$. Thus, the total energy consumption of the found path is $\sum_{i=1}^{k} \mathbf{c}\left(x_{i}\right) \leq$ $k \mathbf{c}(r)$. This implies that the LEARN routing protocol finds the path whose energy consumption is at most $2\left\lceil\frac{1}{1-2 \sin \frac{\alpha}{2}}\right\rceil$ times of the optimum. This finishes the proof.

Notice that the above theorem applies to any general energy model $\mathbf{c}(\|u v\|)$, where $\mathbf{c}(x)$ is a non-decreasing function on $x$, and the energy mileage $\frac{x}{\mathbf{c}(x)}$ is an increasing function when $x<\mathbf{r}_{0}$. Here $\mathbf{r}_{0}$ is the value such that $\frac{\mathbf{r}_{0}}{\mathbf{c}\left(\mathbf{r}_{0}\right)}=\max _{x} \frac{x}{\mathbf{c}(x)}$.

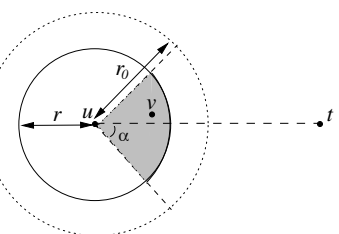

(a) Case 1: $\mathbf{r}_{0} \geq r$

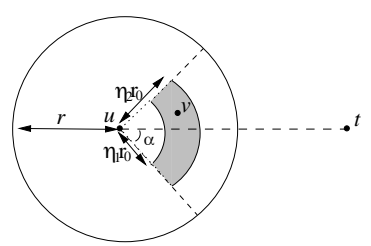

(b) Case 2: $\mathbf{r}_{0}<r$
Fig. 2. Illustrations of the proof of power efficiency: two cases when LEARN routing selects a node inside the restricted region.

We then prove the energy efficiency of LEARN routing protocol for the case when $\mathbf{r}_{0}<r$ and $\mathbf{r}_{0}>0$. See Figure 2 (b) for illustration. In this case, we need an additional requirement that the energy cost $\mathbf{c}(x)$ is smooth around $\mathbf{r}_{0}$, i.e., there exists 
a function $f()$ such that $\mathbf{c}\left(a \mathbf{r}_{0}\right) \leq f(a) \cdot \mathbf{c}\left(\mathbf{r}_{0}\right)$ for any constant $a$ in certain range defined by $\eta_{1} \leq a \leq \eta_{2}$.

Theorem 3: When LEARN routing indeed finds a path $\mathbf{P}_{L E A R N}(s, t)$ from the source $s$ to the target $\mathbf{t}$ and $\mathbf{r}_{0}<r$, the total energy consumption of the found path is within a constant factor of the optimum.

Proof: Notice that when $\mathbf{r}_{0}<r$, our LEARN routing protocol will select a forwarding neighbor $v$ for an intermediate node $u$ such that $\|v u\| \leq \mathbf{r}_{0}$ and $\|t-v\|$ is minimized. Consider any path $v_{0} v_{1} v_{2} \cdots v_{k-1} v_{k}$ found by our routing protocol to connect source node $s=v_{0}$ and destination node $\mathbf{t}=v_{k}$. Let $x_{i}=\left\|v_{i-1} v_{i}\right\|$. Notice that since $\mathbf{r}_{0}<r$, then for any path connecting $v_{0}$ and $v_{k}$, its energy consumption is at least $\frac{\left\|v_{0}-v_{k}\right\|}{\mathbf{r}_{0}} \mathbf{c}\left(\mathbf{r}_{0}\right)$. We will show that the path found by LEARN is within a constant factor of this. Theorem 1 implies that $\sum_{i=1}^{k} x_{i} \leq \frac{1}{1-2 \sin \frac{\alpha}{2}}\left\|v_{0}-v_{k}\right\|$.

When $\eta_{1} \mathbf{r}_{0} \leq x_{i} \leq \eta_{2} \mathbf{r}_{0}$ for $1 \leq i \leq k$, the total energy consumption is $\sum_{i=1}^{k} \mathbf{c}\left(x_{i}\right) \leq f\left(\eta_{2}\right) \cdot k \cdot \mathbf{c}\left(\mathbf{r}_{0}\right)$. Notice that $\sum_{i=1}^{k} x_{i} \leq \frac{1}{1-2 \sin \frac{\alpha}{2}}\left\|v_{0}-v_{k}\right\|$ implies $k \leq \frac{\left\|v_{0}-v_{k}\right\|}{\left(1-2 \sin \frac{\alpha}{2}\right) \eta_{1} \mathbf{r}_{0}}$. Then ratio of the energy consumed by this path over the optimum energy consumption is at most $\frac{f\left(\eta_{2}\right) \cdot k \cdot \mathbf{c}\left(\mathbf{r}_{0}\right)}{\frac{\left\|v_{0}-v_{k}\right\|}{\mathbf{r}_{0}} \mathbf{c}\left(\mathbf{r}_{0}\right)} \leq$

$\frac{f\left(\eta_{2}\right)}{\left(1-2 \sin \frac{\alpha}{2}\right)}$. Notice that for nodes produced by Poisson process with rate $n$ over a compact and convex region, the probability that we cannot find such a neighbor $v$ is $e^{-n \cdot \alpha\left(\eta_{2}^{2}-\eta_{1}^{2}\right) \mathbf{r}_{0}{ }^{2}}$, which clearly goes to 0 as $n$ increases. In other words, when $n$ is sufficiently large, our routing protocol will find a path where each link has length within range $\left[\eta_{1} \mathbf{r}_{0}, \eta_{2} \mathbf{r}_{0}\right]$. This finishes the proof.

For example, for the energy model $\mathbf{c}(x)=x^{2}+c$ with $\eta_{1}=1 / 2$ and $\eta_{2}=2$, we have $f\left(\eta_{2}\right)=5 / 2$. Thus, our routing algorithm finds a path whose energy consumption is at most $\frac{5}{1-2 \sin \frac{\alpha}{2}}$ times of optimum when $\mathbf{r}_{0}<r$.

\section{AsYMPTOTIC TRANSMISSION RANGE FOR LEARN}

This section is devoted to study the asymptotic transmission range for LEARN routing method. In any greedy routing method, the packet may be dropped by some intermediate node $u$ before it reaches the destination $\mathbf{t}$ when node $u$ could not find any of its neighbors that is "better" than itself. Thus, to ensure that the routing is successful for every pair of possible source and destination nodes, each node in the network should have a sufficiently large transmission range such that each intermediate node $u$ will always find a better neighbor. Assume that $V$ is the set of all wireless nodes in the network and each wireless node has a transmission range $r$. Then the physical communication network is modeled by a unit disk graph $G(V, r)$, where two nodes $u$ and $v$ are connected in $G(V, r)$ if and only if their Euclidean distance is at most $r$. A routing method $\mathcal{A}$ is successful over a network $G$ if the routing method $\mathcal{A}$ can find a path for any pair of source and destination nodes. Given a routing method $\mathcal{A}$ and a set of wireless nodes $V$, we define the critical transmission range, denoted as $\rho_{\mathcal{A}}(V)$, for successful routing of $\mathcal{A}$ over $V$ as the minimum transmission range $r$ such that the routing method $\mathcal{A}$ over the network $G(V, r)$ is successful. The subscript $\mathcal{A}$ will be omitted from $\rho_{\mathcal{A}}(V)$ if it is clear from the context.

Previously, several studies (e.g. [18]-[21]) focused on the critical transmission range for certain network properties such as connectivity, $k$-connectivity, and coverage. Traditionally it is assumed that the network nodes are represented by a Poisson point process of density $n$, denoted as $\mathcal{P}_{n}$, over a unit area disk or square; or by random point process of $n$ nodes, denoted as $\mathcal{R}_{n}$, over a unit area disk or square. It was proved in [18], [20] that it is asymptotic almost sure that the network $G\left(\mathcal{P}_{n}, r_{n}\right)$ (also $G\left(\mathcal{R}_{n}, r_{n}\right)$ ) is connected when $n \pi r_{n}^{2}=\beta \ln n$ for any constant $\beta>1$. Here an event is said to be asymptotic almost sure (abbreviated by a.a.s.) if it occurs with a probability converges to one as $n \rightarrow \infty$. It was also shown in [20], [22] that this bound is tight.

Surprisingly, there is not much study for the critical transmission range for certain routing methods, except a recent result [23] for traditional greedy routing [9]. Obviously, for traditional greedy routing, the critical transmission range $\rho(V)$ for successful routing is $\max _{u, v} \min _{w \in \mathbf{L}(u, v)}\|w-u\|$ where lune $\mathbf{L}(u, v)$ is the intersection of two disks centered at $u$ and $v$ respectively using $\|u-v\|$ as radius. It was proved in [23] that $\rho\left(\mathcal{P}_{n}\right)=\sqrt{\frac{\beta_{0} \ln n}{\pi n}}$ a.a.s. for $\beta_{0}=1 /\left(\frac{2}{3}-\frac{\sqrt{3}}{2 \pi}\right) \simeq 1.6^{2}$ and Poisson point process $\mathcal{P}_{n}$ of density $n$ over a convex compact region $\Omega$ with unit area and bounded curvatures.

It is easy to show that, given a set of nodes $V$ already distributed in a region $\Omega$, the critical transmission range $\rho(V)$ for successful routing by restricted greedy routing LEARN is $\max _{u, v} \min _{w: \angle w u v \leq \alpha}\|w-u\|$ where $\alpha$ is the parameter used by LEARN. When the destination node is fixed, say node $\mathbf{t}$, the critical transmission range will be $\max _{u} \min _{w: \angle w u t \leq \alpha} \| w-$ $\mathbf{t} \|$. Here we prove a similar result as in [23] for our restricted greedy routing method LEARN. We also assume that the network nodes are given by a Poisson point process $\mathcal{P}_{n}$ of density $n$ over a convex compact region $\Omega$ with unit area and bounded curvatures.

Theorem 4: The LEARN routing (with parameter $\alpha<\frac{\pi}{3}$ ) will find a path from the source to the target asymptotically almost surely when the transmission range $r_{n}$ satisfies $n \pi r_{n}^{2}=$ $\beta \ln n$ for any constant $\beta>\beta_{0}=\frac{\pi}{\alpha}$.

Proof: To prove this, it is sufficient to show that when each node has a transmission range $r_{n}$ satisfying the above condition, for every node $u$ and every node $v$ there is always a node $w \in \mathcal{P}_{n}$ such that $\angle w u v \leq \alpha$ and $\|w-u\| \leq r_{n}$, i.e., any intermediate node $u$ can find a "better" neighbor $w$ towards the destination node $v$.

Given a point distribution $\mathcal{P}_{n}$, let $\mathcal{S}\left(\mathcal{P}_{n}, r_{n}\right)$ be the minimum number of such neighboring nodes $w$ that can be chosen by any intermediate node $u$ for any possible destination $v$. As proved in [23], it suffices to prove that the cardinality $\left|\mathcal{S}\left(\mathcal{P}_{n}, r_{n}\right)\right|>0$. We actually will show a much stronger result that $\mathcal{S}\left(\mathcal{P}_{n}, r_{n}\right) \geq \frac{1}{2} \mathcal{L}\left(\frac{\beta}{\beta_{2}}\right) \ln n$ for any constant $\beta_{0}<\beta_{2}<\beta$. Here $\mathcal{L}(x)$ is defined in [23] as $\mathcal{L}(x)=x \phi^{-1}(1 / x)$ for $x>0$ and $\phi(x)=1+x \ln x-x$ for $x>0$.

Notice that, given a node $u$, the area that node $u$ can choose 
its neighbor to forward data for a given destination $\mathbf{t}$ is a sector of disk $\mathbf{D}\left(u, r_{n}\right)$ with angle $2 \alpha$. Here $\mathbf{D}(u, r)$ denotes the disk centered at node $u$ with radius $r$. We use $\mathbf{Y}$ to denote such sector. Let $d$ denote the diameter of this sector Y. Clearly $d=r_{n}$ when $\alpha \leq \frac{\pi}{6}$, and $d=2 \sin \alpha \cdot r_{n}$ when $\frac{\pi}{6} \leq \alpha<\frac{\pi}{3}$.

Assume that the space is partitioned into grids (quadrates) of side length $\eta$, which we call it $\eta$-tessellation of space. In this paper, we will consider $\epsilon d$-tessellation, where $\epsilon$ is a constant to be specified later. A polyquadrate is defined as the set of quadrates that intersect with a convex and compact region, e.g., Y. Notice that when the grid-partition shifts, we will have different polyquadrates for the fixed region Y. A polyquadrate in a $\eta$-tessellation is said to have a span $s$ if it can be contained in a square of side-length $s \cdot \eta$. We are only interested in polyquadrates that has span at most $\frac{1}{\epsilon}$ and area at least a certain fraction of $\pi r_{n}^{2}$. Assume that, given $\mathbf{Y}$, there are $I_{n}$ different such polyquadrates that are completely contained inside, with span at most $\frac{1}{\epsilon}$ and area at least $\delta_{1} \pi r_{n}^{2}$. Here $\delta_{1}$ is a constant to be specified later. For $i$ th such polyquadrate, let $X_{i}$ denote the number of nodes of $V$ contained inside. Also assume that there are $I_{n}^{\prime}$ different such polyquadrates that intersect the boundary of $\mathbf{Y}$ with span at most $\frac{1}{\epsilon}$ and area at least $\delta_{2} \pi r_{n}^{2}$. Here $\delta_{2}$ is a constant to be specified later. For $i$ th such polyquadrate, let $X_{i}^{\prime}$ denote the number of nodes of $V$ contained inside. According to Lemma 4 of [23], we have $I_{n}=O\left(\left(\frac{1}{\epsilon d}\right)^{2}\right)=O\left(\frac{n}{\ln n}\right), I_{n}^{\prime}=O\left(\frac{1}{\epsilon d}\right)=O\left(\sqrt{\frac{n}{\ln n}}\right)$. Furthermore, $X_{i}$ are Poisson random variables with rate at least $\delta_{1} \pi r_{n}^{2} \cdot n=\beta \delta_{1} \ln n$. Similarly, $X_{i}^{\prime}$ are Poisson random variables with rate at least $\delta_{2} \pi r_{n}^{2} \cdot n=\beta \delta_{2} \ln n$. Then according to Lemma 6 of [23], we have $\min _{i=1}^{I_{n}} X_{i}>\mathcal{L}\left(\beta^{\prime}\right) \ln n$ a.a.s. for any $1<\beta^{\prime}<\delta_{1} \beta$, and $\min _{i=1}^{I_{n}^{\prime}} X_{i}^{\prime}>\frac{1}{2} \mathcal{L}\left(2 \beta^{\prime \prime}\right) \ln n$ a.a.s. for any $1<\beta^{\prime \prime}<\delta_{2} \beta$. Thus, we have a.a.s. $\min \left(\min _{i=1}^{I_{n}} X_{i}, \min _{i=1}^{I_{n}^{\prime}} X_{i}^{\prime}\right) \geq \min \left(\mathcal{L}\left(\beta^{\prime}\right), \frac{1}{2} \mathcal{L}\left(2 \beta^{\prime \prime}\right)\right) \ln n$ for any $1<\beta^{\prime}<\delta_{1} \beta$, and $1<\beta^{\prime \prime}<\delta_{2} \beta$.

To prove the theorem, it is sufficient to show that $\mathcal{S}\left(\mathcal{P}_{n}, r_{n}\right) \geq \min \left(\min _{i=1}^{I_{n}} X_{i}, \min _{i=1}^{I_{n}^{\prime}} X_{i}^{\prime}\right)$. In other words, we only need to show that for any $\mathbf{Y}$, it

1) either contains a polyquadrate $P$ has span at most $\frac{1}{\epsilon}$ and area at least $\delta_{1} \pi r_{n}^{2}$ when $\mathbf{Y}$ is inside $\Omega$;

2) or contains a polyquadrate $P^{\prime}$ has span at most $\frac{1}{\epsilon}$ and area at least $\delta_{1} \pi r_{n}^{2}$ when $\mathbf{Y}$ intersects boundary of $\Omega$.

First consider the case when $\mathbf{Y}$ is contained inside $\Omega$. For the polyquadrate $P$, we consider the induced polyquadrate (denoted by $P_{-\sqrt{2} \epsilon d}$, formed by all quadrates of $P$ that intersect with region $\mathbf{Y}_{-\sqrt{2} \epsilon d}$. Here $\mathbf{Y}_{-x}$ denotes the region of $\mathbf{Y}$ that are of distance at least $x$ from the boundary of $\mathbf{Y}$. The span of polyquadrate $P_{-\sqrt{2} \epsilon d}$ is at most $\left\lceil\frac{d-2 \sqrt{2} \epsilon d}{\epsilon d}\right\rceil+1<\frac{1}{\epsilon}$. The area of the polyquadrate $P_{-\sqrt{2} \epsilon d}$ is at least the area of $\mathbf{Y}_{-\sqrt{2} \epsilon d}$. Notice that $\left|\mathbf{Y}_{-\sqrt{2} \epsilon d}\right|=\alpha\left(r_{n}-2 \sqrt{2} \epsilon d\right)^{2}$. Thus, it is sufficient to require that $\left|\mathbf{Y}_{-\sqrt{2} \epsilon d}\right|=\alpha\left(r_{n}-2 \sqrt{2} \epsilon d\right)^{2} \geq \delta_{1} \pi r_{n}^{2}$.

We then consider the case that $\mathbf{Y}$ intersects the boundary of $\Omega$. Then let $\mathbf{Y}^{\prime}$ be the part that are fully contained inside $\Omega$. Similarly, we consider the polyquadrate $P_{-\sqrt{2} \epsilon d}^{\prime}$ induced by $\mathbf{Y}_{-\sqrt{2} \epsilon d}^{\prime}$, i.e., the quadrates that are contained inside $\Omega$ and $\mathbf{Y}$. Clearly, the span of this polyquadrate is also at most $\frac{1}{\epsilon}$. It is not difficult to show that the area of $\mathbf{Y}^{\prime}$ is at least $\frac{1}{2}$ of the area of $\mathbf{Y}$. Thus, the area $\left|P_{-\sqrt{2} \epsilon d}^{\prime}\right|$ of polyquadrate $P_{-\sqrt{2} \epsilon d}^{\prime}$ is at least $\left|P_{-\sqrt{2} \epsilon d}^{\prime}\right| \geq\left|\mathbf{Y}_{-\sqrt{2} \epsilon d}^{\prime}\right| \geq \frac{1}{2}\left|\mathbf{Y}_{-\sqrt{2} \epsilon d}\right|$. Thus, it is sufficient to require that $\frac{1}{2}\left|\mathbf{Y}_{-\sqrt{2} \epsilon d}\right|=\frac{1}{2} \alpha\left(r_{n}-2 \sqrt{2} \epsilon d\right)^{2} \geq \delta_{2} \pi r_{n}^{2}$.

In summary, we require the following conditions about the parameters $\delta_{1}, \delta_{2}, \epsilon$ :

$$
\left\{\begin{aligned}
\beta \delta_{1} & >1, \\
\beta \delta_{2} & >1, \\
\alpha\left(1-2 \sqrt{2} \epsilon \frac{d}{r_{n}}\right)^{2} & \geq \delta_{1} \pi, \\
\alpha\left(1-2 \sqrt{2} \epsilon \frac{d}{r_{n}}\right)^{2} & \geq 2 \delta_{2} \pi .
\end{aligned}\right.
$$

Clearly, we can choose $\delta_{2}=\frac{1}{2} \delta_{1}$. Notice that we defined $\beta_{0}=\frac{\pi}{\alpha}$. Thus, it is equivalent to require that

$$
\left\{\begin{aligned}
\beta \delta_{1} & >1 \\
\left(1-2 \sqrt{2} \epsilon \frac{d}{r_{n}}\right)^{2} & \geq \delta_{1} \beta_{0} .
\end{aligned}\right.
$$

This clearly has a solution when $\beta>\beta_{0}$. For example, we can select a constant $\beta_{1}$ such that $\beta_{0}<\beta_{1}<\beta$ and let $\delta_{1}=\frac{1}{\beta_{1}}$. Then $\epsilon=\frac{1-\sqrt{\beta_{0} / \beta_{1}}}{2 \sqrt{2} d_{0}}$. Here $d_{0}=\frac{d}{r_{n}}$, which is 1 when $\alpha \leq \frac{\pi}{6}$ and is $2 \sin \alpha$ when $\frac{\pi}{6} \leq \alpha<\frac{\pi}{3}$. In this case, we can choose $\beta_{2}$ such that $\beta_{0}<\beta_{1}<\beta_{2}<\beta$ and set $\beta^{\prime}=\frac{\beta}{\beta_{2}}$ and $\beta^{\prime \prime}=\beta^{\prime} / 2$. Then we have $\mathcal{S}\left(\mathcal{P}_{n}, r_{n}\right) \geq \frac{1}{2} \mathcal{L}\left(\frac{\beta}{\beta_{2}}\right) \ln n$.

Notice that when $r_{n}=\sqrt{\frac{\beta \ln n}{\pi n}}$ for some $\beta>\beta_{0}=\frac{\pi}{\alpha}$, the probability that an intermediate node $u$ cannot find a forwarding node $w$ to a destination $\mathbf{t}$ is $e^{-n \alpha r_{n}^{2}}$, i.e., the probability that the sector does not contain any node. Since the path from any source node to any destination node contains at most $n$ hops, the probability that LEARN routing protocol is successful is at least $\left(1-e^{-n \alpha r_{n}^{2}}\right)^{n}=\left(1-n^{-\frac{\beta}{\beta_{0}}}\right)^{n}>$ $1-\frac{1}{n^{\beta / \beta_{0}-1}}$, which goes to 1 as $n \rightarrow \infty$.

Notice that in the above proof, we can find $\epsilon, \delta_{1}$ and $\delta_{2}$ only if $\beta>\beta_{0}=\frac{\pi}{\alpha}$. It is thus natural to conjecture that $\beta_{0}$ is the threshold value. It is indeed true based on following:

Theorem 5: The LEARN routing (with parameter $\alpha<\frac{\pi}{3}$ ) will not be able to find a path from the source to the target asymptotically almost surely when the transmission range $r_{n}$ satisfies $n \pi r_{n}^{2}=\beta \ln n$ for any constant $\beta<\beta_{0}=\frac{\pi}{\alpha}$.

Proof: We basically will show that, a.a.s., there are two nodes $u$ and $v$ such that we cannot find a node $w$ for forwarding by node $u$, i.e., there does not exist node $w$ with $\angle w u v \leq \alpha$ and $\|w-u\| \leq r_{n}$. Recall that $r_{n}=\sqrt{\frac{\beta \ln n}{\pi n}}$ for $\beta<\beta_{0}$. Again we partition the space using grids, where each grid has side-length $\eta r_{n}$ for a constant $0<\eta$ to be specified later. Then it is easy to show that the number of cells, denoted by $I_{n}$ here, that are fully contained inside the compact and convex region $\Omega$ with unit area is $\Theta\left(\frac{1}{\eta^{2} r_{n}^{2}}\right)=\Theta\left(\frac{n}{\ln n}\right)$. Let $E_{u, v}$ denote the event that no forwarding node $w$ (with $\angle w u v \leq \alpha$ and $\|w-u\| \leq r_{n}$ ) exists for node $u$ to reach node $v$. Then to prove our theorem, it is equivalent to prove that the probability at least one of the event $E_{u, v}$ happens asymptotically almost sure, i.e., $1-\operatorname{Pr}$ (none of event $E_{u, v}$ happens). Clearly, the events $E_{u, v}$ are not independent for all pairs $u$ and $v$. We will consider a special subset of events that are independent. Consider any cell produced by the grid partition that are contained inside $\Omega$. See Figure 3 (a) for an illustration. 
For each cell, we draw a shaded square with side-length $(\eta-2(1+\delta)) r_{n}$ and it is of distance $(1+\delta) r_{n}$ to the boundary of the cell. We only consider the case when node $u$ is located in the shaded square as in Figure 3 (a). We also restrict the node $v$ to satisfy that $r_{n}<\|u-v\| \leq(1+\delta) r_{n}$, i.e., in the torus area in Figure 3 (b). Clearly, node $v$ will also be inside this cell, and the shaded sector area (see Figure 3 (b)) where the possible forwarding node could locate is also inside this cell. Thus, events $E_{u_{1}, v_{1}}$ and $E_{u_{2}, v_{2}}$ will be independent if $u_{1}$ and $u_{2}$ are selected as above from different cells.

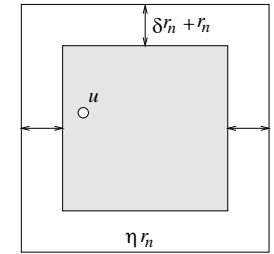

(a) selection of $u$

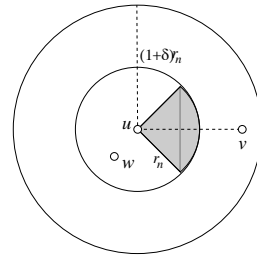

(b) no forwarding node
Fig. 3. Illustrations of the proof of lower bound: (a) a cell and the area where we will select a node $u$, (b) the event that node $u$ cannot find a forwarding node $w$ to reach a node $v$.

Then for each cell $i$, we compute the probability that the event $E_{u_{i}, v_{i}}$ happens, where $u_{i}$ is selected from the shaded square of cell $i$ and $v_{i}$ is selected such that $r_{n}<$ $\left\|v_{i}-u_{i}\right\| \leq(1+\delta) r_{n}$. Recall that for any region with area $A$, the probability that this region is empty of any nodes (for Poisson process with rate $n$ ) is $e^{-n A}$. Clearly, the probability that node $u_{i}$ exists is $1-e^{-n(\eta-2-2 \delta) r_{n}^{2}}$ since the shared square has area $(\eta-2-2 \delta) r_{n}^{2}$; the probability that node $v_{i}$ exists is $1-e^{-n\left(\delta^{2}+2 \delta\right) r_{n}^{2}}$ since the torus has area $\left(\delta^{2}+2 \delta\right) r_{n}^{2}$. Given node $u_{i}$ and $v_{i}$, the probability that event $E_{u_{i}, v_{i}}$ happens is $e^{-n \alpha r_{n}^{2}}=e^{-\beta / \beta_{0} \ln n}=n^{-\beta / \beta_{0}}$. Consequently, event $E_{u, v}$ happens for some node pairs $u$ and $v$ is

$$
\begin{array}{r}
\operatorname{Pr}\left(E_{u_{i}, v_{i}}\right) \geq\left(1-e^{-n(\eta-2-2 \delta) r_{n}^{2}}\right)\left(1-e^{-n\left(\delta^{2}+2 \delta\right) r_{n}^{2}}\right) n^{-\beta / \beta_{0}} \\
=\left(1-n^{-\beta(\eta-2-2 \delta) / \pi}\right)\left(1-n^{-\beta\left(\delta^{2}+2 \delta\right) / \pi}\right) n^{-\beta / \beta_{0}} .
\end{array}
$$

Thus, the probability that the LEARN routing fails to find a path for some pair of source and destination nodes is

$$
\begin{aligned}
& \operatorname{Pr}\left(\text { at least one of events } E_{u, v} \text { happens }\right) \\
\geq & \operatorname{Pr}\left(\text { at least one of events } E_{u_{i}, v_{i}} \text { happens }\right) \\
= & 1-\operatorname{Pr}\left(\text { none of event } E_{u_{i}, v_{i}} \text { happens }\right) \\
= & 1-\left(1-\operatorname{Pr}\left(E_{u_{i}, v_{i}}\right)\right)^{I_{n}} \\
= & 1-e^{I_{n} \cdot \ln \left(1-\operatorname{Pr}\left(E_{u_{i}, v_{i}}\right)\right)} \geq 1-e^{-I_{n} \cdot \operatorname{Pr}\left(E_{u_{i}, v_{i}}\right) .}
\end{aligned}
$$

Notice that $I_{n} \cdot \operatorname{Pr}\left(E_{u_{i}, v_{i}}\right)=\Theta\left(\frac{n}{\ln n}\right)\left(1-n^{-\beta(\eta-2-2 \delta) / \pi}\right)(1-$ $\left.n^{-\beta\left(\delta^{2}+2 \delta\right) / \pi}\right) n^{-\beta / \beta_{0}} \simeq \frac{n^{1-\beta / \beta_{0}}}{\ln n}$, which goes to $\infty$ as $n \rightarrow$ $\infty$ when $\beta<\beta_{0}, \eta-2-2 \delta>0$, and $\delta>0$. This can be easily satisfied, e.g., $\delta=1, \eta=5$. Thus, $\lim _{n \rightarrow \infty} 1-$ $e^{-I_{n} \cdot \operatorname{Pr}\left(E_{u_{i}, v_{i}}\right)}=1$.

Notice that above results assume that the deployment region $\Omega$ has a unit area. Generally, we will often have a convex and compact region $\Omega$ with area $\mathcal{D}$, and the transmission range $r$ could be fixed (or dynamically changed based on node density). Assume again that the network nodes are produced by a Poisson process with rate $n$ (i.e., the expected number of nodes in a unit area is $n$, thus the total number of deployed nodes in the area is $n \mathcal{D}$ ). Then by a proper scaling of the distance unit, we have the following theorem

Theorem 6: When the transmission range $r_{n}$ and the Poisson process rate $n$ satisfy that $n \pi r_{n}^{2}=\beta \cdot \ln (\mathcal{D} \cdot n)$ for any $\beta>\beta_{0}$, our LEARN routing protocol will successfully route the data a.a.s.. When $n \pi r_{n}^{2}=\beta \cdot \ln (\mathcal{D} \cdot n)$ for any $\beta<\beta_{0}$, our LEARN protocol will not be able to route the data a.a.s..

So far, we mainly concentrated on the routing LEARN. Notice that the critical transmission range of our LEARN$\mathrm{G}$ routing protocol will be exactly same as the traditional greedy routing [9] method since at last we use the greedy routing to find the forwarding node if LEARN fails. There are a number of other localized routing methods developed already and many to be developed in the future. We thus would like to know the general critical transmission range for successful routing by any localized routing method $\mathcal{A}$. We then generalize the above theorems and conclude that,

Theorem 7: For a general localized routing method $\mathcal{A}$, the critical transmission range is $\rho_{\mathcal{A}}\left(\mathcal{P}_{n}\right)=\sqrt{\frac{\beta_{\mathcal{A}} \ln (\mathcal{D} \cdot n)}{n \pi}}$. Here $\beta_{\mathcal{A}}$ is the ratio of the area of the disk centered at an intermediate node $u$ with radius $r_{n}$ over the area of the forwarding region in this disk from where the intermediate node $u$ can choose its next neighbor $w ; \mathcal{D}$ is the area of the convex and compact deployment region; and $n$ the rate of the Poisson point process. We require that the forwarding region of any intermediate node $u$ for any target node $\mathbf{t}$ is convex and compact and at least a constant fraction of the forwarding region is contained inside the deployment region $\Omega$.

Notice that the above theorem not only applies to the routing method, it also applies to the critical range for the connectivity of the network in which $\beta_{\mathcal{A}}=1$. This is based on the following observation: a network, formed by a set $V$ of $n$ nodes and each node has a transmission range $r_{n}$, is connected if and only if the routing method $\mathcal{H}$ that uses the path with the minimum hop number can successfully find a path for every pair of source and destination nodes. For this special routing method $\mathcal{H}$, clearly the area to find the forwarding node $w$ by a node $u$ is the disk $\mathbf{D}\left(u, r_{n}\right)$, i.e., $\beta_{\mathcal{H}}=1$.

So far, we assumed that the link is always reliable and the nodes are always awake. This is always an ideal case. To capture the practical aspects of wireless networks, we assume that a wireless link $u v$ is reliable with a constant probability $p_{1}>0$ and each node is awake with a constant probability $p_{2}>0$. Similarly we can show that that the critical transmission range for a successful routing by a general routing method $\mathcal{A}$ is $\rho_{\mathcal{A}}\left(\mathcal{P}_{n}\right)=\sqrt{\frac{\beta_{\mathcal{A}} \ln (\mathcal{D} \cdot n)}{n \pi p_{1} p_{2}}}$.

\section{PRACTICAL IMPLEMENTATION AND IMPROVEMENTS}

\section{A. Face Routing After LEARN Fails}

It is well-known in the literature that greedy routing may fail to delivery the packets in certain circumstances. Face routing is then often used (e.g., [9], [10], [12]) to get out of the 
local minimum encountered in the greedy routing. For face routing based on a certain planar graph $H$, actually, some improvements can also be made to reduce the total energy consumption. Assume that $u v$ and $v w$ are links in the planar graph $H$ and face routing selects both links $u v$ and $v w$ for routing. If $u$ and $w$ are within the transmission range of each other (clearly they may not be neighbors in $H$ ), we can short-cut the route by replacing $u v$ and $v w$ with the direct link $u w$ only. Similar improvement can be made till no such improvement exists. This simple short-cut will reduce the hop number of the found path. Since here we want to design energy efficient routing protocols, we can use a new criterion to decide whether to do short-cut: we do short-cut only if the energy consumption of link $u w$ is less than the total energy consumption of links $u v$ and $v w$.

\section{B. Mobile Networks}

So far, all above studies have assumed a static random network. A static random network can model static sensor networks well, where sensors are distributed randomly and will be static after deployment. However, in certain applications, the wireless nodes may be mobile during the communication. Notice that for static networks, greedy routing will terminate after a finite time because at any intermediate node $u$, we will always make a positive movement towards the target $\mathbf{t}$ if any intermediate node $u$ finds a forwarding node. Thus, the path from the source to the target involves at most $n$ nodes for a network with $n$ wireless terminals. The above argument will not extend directly to the case when the intermediate nodes are mobile. Since forwarding packets, receiving packets, and processing packets will incur some delay, a mobile intermediate node $u$ will often have different locations at different stage of the forwarding packets. It could happen that the node $u$ may not move toward the destination node $\mathbf{t}$. Consequently, the absolute distance of the selected next-hop node $w$ to the target $\mathbf{t}$ may be longer than what was already achieved by some previous node on the route, even by node $u$.

When nodes are mobile, instead of face routing, we will let each node hold the packets for a short-time (called holding time) when it cannot find a forwarding neighbor initially, in the hope that it will find another node that is closer to target node during this holding time when all nodes are moving. If no such node exists after this holding time interval, this intermediate node will drop the packets.

By assuming (1) the destination node $\mathbf{t}$ is static, (2) the delay $T_{0}$ from the moment an intermediate node $u$ is selected as the forwarding node to the moment that node $u$ selects the nexthop node $w$ for forwarding (plus the holding time mentioned above) is bounded by constant, and (3) the maximum distance that this intermediate node $u$ can travel during this time interval $T_{0}$ is bounded by a constant $\ell$, we can prove that LEARN routing protocol will terminate in mobile networks with high probability. Notice that our LEARN routing protocol will terminate when an intermediate node $u$ cannot find any forwarding node $w$ after it received a forwarding request but before the holding time expires. When $u$ can find a forwarding node $w$, we can show that the expected forwarding distance by node $w$ is at least $\Theta\left(r_{n}\right)$ for any possible feasible location of $u$ and $w$. Observe that the Euclidean distance between any pair of source and target is at most $O\left(n \cdot r_{n}\right)$ (otherwise the network will not be connected). Thus, the LEARN routing protocol will terminate after at most $\Theta(n)$ forwardings. However, this does not directly implies that it will find the path from any pair of nodes asymptotically almost surely. The reason is that now the number of hops of the path could be much longer than the static case, thus, the probability that the routing is successful becomes smaller. We leave it as a future work to compute the critical transmission range for mobile networks: the relations between the critical transmission range for successful routing and the moving distance $\ell$.

\section{Other Issues}

In our LEARN routing protocol, the neighbor node $v$ is selected after the destination node $\mathbf{t}$ is known. Here we propose a variation of LEARN routing protocol, called LEARN-Y, in which each intermediate node $u$ will pre-select a constant number of candidate forwarding neighbors $v$. It essentially uses a structure similar to Yao structure [24]. A node $u$ partitions the region surrounded $u$ into $k$ equal-sized cones centered at $u$. At each cone $i$, node $u$ selects the neighboring node $v_{i}$ that is closest to $\mathbf{r}_{0}$. When node $u$ needs to forward packets for a destination node $\mathbf{t}$, it then forward it to the neighbor $v_{i}$ that is at the same cone as $\mathbf{t}$. It is easy to show that the number of links used for routing is at most $k \cdot n$. Similarly, we can also prove that LEARN-Y routing protocol will successfully route the data if $r_{n}=\sqrt{\frac{\beta \ln n}{\pi n^{2}}}$ for any $\beta>k$, and the found path is energy efficient whenever $k>6$.

Notice that so far we only concentrated on the energy consumption of the path. In practice, sometimes, it may be more interesting to find a path that can support the maximum throughput per unit energy consumption by the network. Thus, instead of selecting link that has the best energy mileage, one possible criterion for selecting best neighbors could be to select a neighbor $v$ such that the following is maximized: $\|u v\| \cdot B(u v) / \mathbf{c}(u v)$. Here $B(u v)$ is the bandwidth of link $u v$ and $\mathbf{c}(u v)$ is the energy consumption of link $u v$. When $B(u v)$ is uniform for all links, this is exactly our LEARN protocol.

\section{Vi. Performance Evaluation}

In this section, we will study the critical transmission range for LEARN and its piratical performance (in terms of delay, energy consumption, and network throughput) via simulation.

\section{A. Critical Transmission Range for LEARN}

We first study the study the critical transmission range for restricted greedy routing LEARN in random networks. In our experiments, we randomly distribute $n$ wireless nodes in a disk with radius 5 unit. Node density $n$ is $50,100,200$, 300,400 , and 500 . The parameter $\alpha$ is $\pi / 4$ or $\pi / 6$. For each choice of $\alpha$ and $n, 1000$ sample of networks is generated, and the critical transmission range is generated for each sample. Figure 4 illustrates the average and 95 percentile critical 
transmission range for our restricted greedy routing LEARN in random networks when $\alpha$ are $\pi / 4$ and $\pi / 6$. It is clear the critical transmission range decreases when the number of nodes increases. And with smaller $\alpha$, LEARN need a larger critical transmission range to guarantee the delivery. Figure 5 shows the detailed distributions of the critical transmission range in random networks with different size when $\alpha=\pi / 4$.

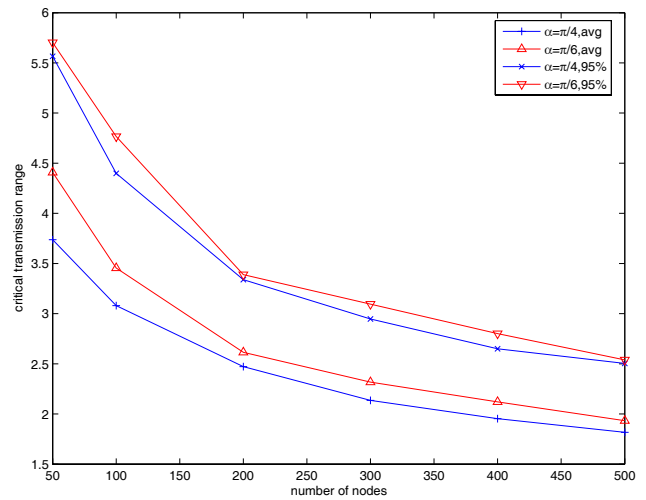

Fig. 4. The average and 95 percentile critical transmission range for LEARN routing in random networks when $\alpha=\pi / 4, \pi / 6$.

\section{B. Network Performance Comparisons}

We then conducted extensive simulations to evaluate the actual routing performance of our algorithm LEARN using QualNet 3.9 simulation tools [25], by comparing it with GPSR [10] and DSR [26]. we randomly deploy a group of wireless nodes to a $1000 \mathrm{~m} \times 1000 \mathrm{~m}$ square area. One node is randomly designated as the sink, and all the other nodes periodically generate data and send to the sink. We adopt IEEE $802.11 \mathrm{~b}$ in physical and MAC layer model. The transmission data rate is set to $2 \mathrm{Mbps}$. The maximum transmission power of every node is $15.0 \mathrm{dBm}$ and receiver sensitivity is $-89.0 \mathrm{dBm}$. We simulate periodical traffic from all sensors to the sink using CBR (Constant Bit Rate) scenario, i.e., each node send 50 rounds data to the sink with 128 bytes each. Qualnet does not provide energy model in standard distribution, while several different energy models are developed by end users. In our simulation, we let each node $u$ adjust transmission range to sufficiently reach the next hop $v$ and assume the energy consumption is $\|u v\|^{2}+C$, where $C=\frac{1}{4} r^{2}$ and $r$ is the maximum transmission range of each node. The values of $\eta_{1}$, $\eta_{2}$ and $\alpha$ are $1 / 2,2$ and $55^{\circ}$ in all simulations.

1) Performance with Static Networks: We first evaluate the routing performance in static scenario, where nodes position does not change during the routings. For our algorithm LEARN, we use the one with the face routing, i.e., LEARNGF. We vary the network size from 30 to 120 and evaluate the average packet delays, energy consumptions and network throughput, as shown in Figure 6 (a)-(c). Given each network size, we generate 50 random topologies to get the average performance. We first compare packet delay of three methods. Here, packet delay is the time cost for one round successful information gathering. When we measure packet delay, we wait until all one-round packets reach the sink. In other words, it is basically the maximum packet delay of all packets in one round. DSR has larger packet delays and consume more energy as expect. Our LEARN algorithm indeed consumes $30 \%$ less energy than GPSR, since we carefully choose next hop to increase energy mileage. The packet delay and network throughput of LEARN is about $96 \%$ of that through GPSR routing similar network performance. Notice that LEARN often uses links that are shorter than the one used by GPSR, and thus its routing path often has more hops than GPSR. The disadvantage of more hops is compensated by generally the improved reliability of shorter links.

2) Performance with Mobile Networks: We then evaluate the performance in mobile scenario, where sink is static and all the other nodes follow random Waypoint mobility model. For random waypoint, a node randomly selects a destination from the physical terrain. It moves to the destination at constant speed, chosen between MOBILITY-WP-MIN-SPEED and MOBILITY-WP-MAX-SPEED (meters/sec). After it reaches its destination, the node stays there for MOBILITY-WPPAUSE time, and the process is repeated (selecting another destination and speed). Here we set MOBILITY-WP-MINSPEED $=0$ meters/second and MOBILITY-WP-PAUSE = 10 seconds, while varying MOBILITY-WP-MAX-SPEED in the range $[10,100]$ meters/second. Here, we do not use face routing for our LEARN, i.e, we test LEARN-G. Figure 6 (d)-(f) show the results. Similarly DSR has larger packet delays and consume more energy as expected. Routing based on LEARN algorithm consumes about $26 \%$ less energy than GPSR, and the packet delays and throughput of LEARN and GPSR are again similar (about 98\%).

For both static networks and mobile networks, we also perform several experiments by varying data reporting intervals. The results are consistent, and ignored due to space limit.

\section{CONCLUSION}

We proposed localized energy aware restricted neighborhood routing protocol LEARN for wireless sensor or ad hoc networks. We theoretically proved that our LEARN routing protocol is energy efficient. We also studied the critical transmission range for the successful packet delivery a.a.s. Our mathematical formulation also extends to any routing protocol in which the region to find the next hop node by an intermediate node is compact and convex. We conducted extensive simulations to study the performance of our LEARN routing compared with GPSR and DSR. To the best of our knowledge, our new localized routing method is the first localized routing protocol that has theoretical guarantee of power efficiency of the generated routes in random networks with high probability.

\section{REFERENCES}

[1] S. Doshi, S. Bhandare, and T. X. Brown, "An on-demand minimum energy routing protocol for a wireless ad hoc network," ACM Mob. Comput. Commun. Rev., vol. 6, no. 3, pp. 50-66, 2002.

[2] K. Kar, M. Kodialam, T. Lakshman, and L. Tassiulas, "Routing for network capacity maximization in energy-constrained ad-hoc networks," in IEEE INFOCOM, 2003. 


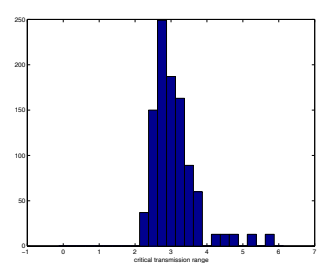

(a) 100 nodes

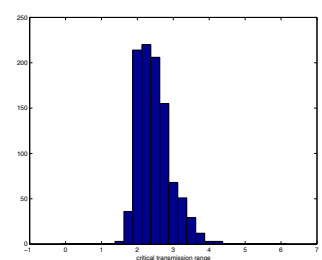

(b) 200 nodes

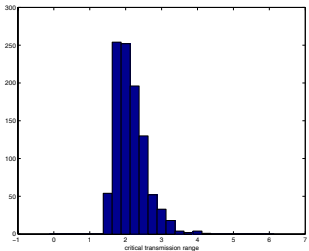

(c) 300 nodes

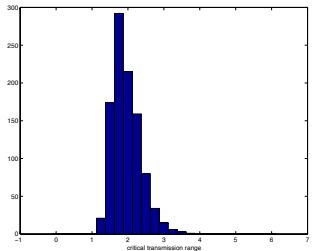

(d) 400 nodes

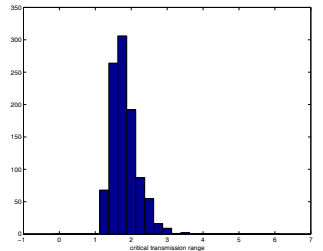

(e) 500 nodes

Fig. 5. The distributions of the critical transmission range for LEARN routing in random networks when $\alpha=\pi / 4$.

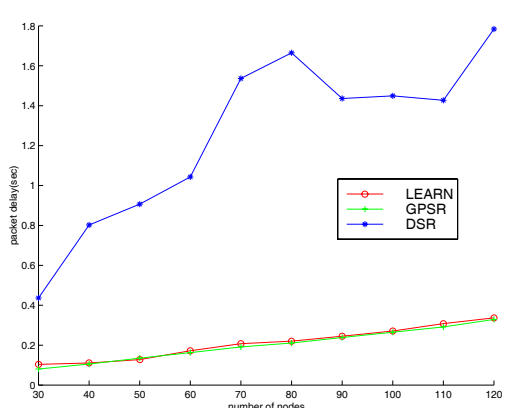

(a) packet delay

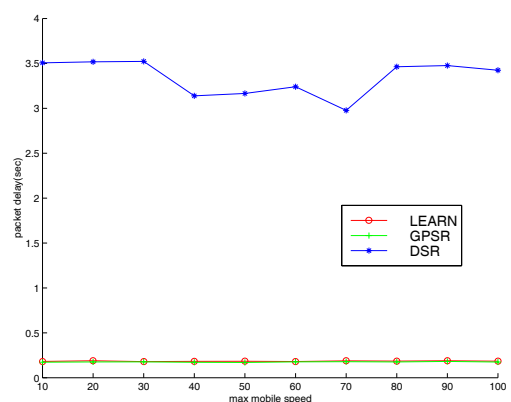

(d) packet delay

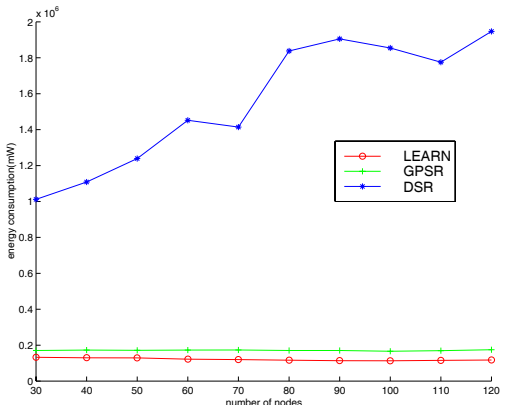

(b) energy consumption

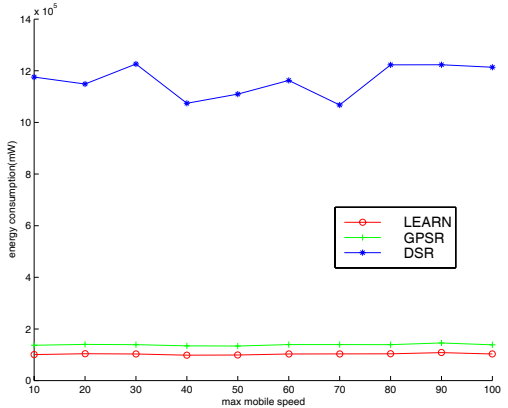

(e) energy consumption

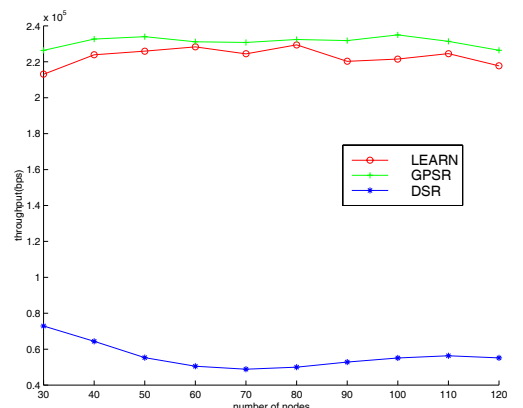

(c) network throughput

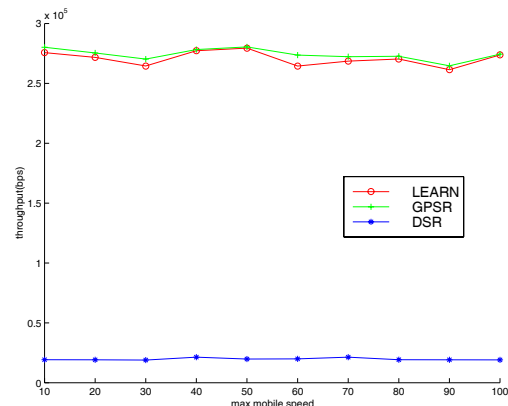

(f) network throughput

Fig. 6. (a)-(c) Performance of static networks with various sizes $(30-120)$. (d)-(f) Performance of mobile networks with various maximum speeds (10 - 100).

[3] J.-H. Chang and L. Tassiulas, "Energy conserving routing in wireless ad-hoc networks," in IEEE INFOCOM, 2000.

[4] Q. Li, J. Aslam, and D. Rus, "Online power-aware routing in wireless ad-hoc networks," in ACM MobiCom, 2001.

[5] Q. Dong, S. Banerjee, M. Adler, and A. Misra, "Minimum energy reliable paths using unreliable wireless links," in ACM MobiHoc, 2005.

[6] T. Melodia, D. Pompili, and I. F. Akyildiz, "Optimal local topology knowledge for energy efficient geographical routing in sensor networks," in IEEE INFOCOM, 2004.

[7] I. Stojmenovic and S. Datta, "Power and cost aware localized routing with guaranteed delivery in wireless networks," Wireless Comm. and Mobile Compt., vol. 4, no. 2, pp. 175-188, 2004.

[8] I. Stojmenovic and X. Lin, "Loop-free hybrid single-path/flooding routing algorithms with guaranteed delivery for wireless networks," IEEE Trans. on Parallel and Distributed Systems, vol. 12, no. 10, 2001.

[9] P. Bose, P. Morin, I. Stojmenovic, and J. Urrutia, "Routing with guaranteed delivery in ad hoc wireless networks," ACM/Kluwer Wireless Networks, vol. 7, no. 6, 2001.

[10] B. Karp and H. Kung, "GPSR: Greedy perimeter stateless routing for wireless networks," in ACM MobiCom, 2000.

[11] F.Kuhn, R.Wattenhofer, A.Zollinger, "Worst-Case Optimal and AverageCase Efficient Geometric Ad-Hoc Routing," in ACM MobiHoc, 2003.

[12] F. Kuhn, R. Wattenhofer, Y. Zhang, and A. Zollinger, "Geometric AdHoc Routing: Of Theory and Practice"," in ACM PODC, 2003.

[13] T. Roosta, "Probabilistic geographic routing in ad hoc and sensor networks," in Proc. of Int. Workshop on Wireless Ad-hoc Networks, 2005.

[14] F. Kuhn, R. Wattenhofer, and A. Zollinger, "Asymptotically optimal geometric mobile ad-hoc routing," in ACM Dial-M, 2002.
[15] E. Kranakis, H. Singh, and J. Urrutia, "Compass routing on geometric networks," in Proc. 11 th Canadian Conf. on Compt. Geometry, 1999.

[16] P. Bose and P. Morin, "Online routing in triangulations," in Proc. of the 10 th Annual Int. Symp. on Algorithms and Computation, 1999.

[17] Y. Wang and X.-Y. Li, "Efficient delaunay-based localized routing for wireless sensor networks,", 2006, submitted for publication.

[18] P. Gupta and P. R. Kumar, "Critical power for asymptotic connectivity in wireless networks," Stochastic Analysis, Control, Optimization and Applications. 1998

[19] X.-Y. Li, P.-J. Wan, Y. Wang, C.-W. Yi, and O. Frieder, "Robust deployment and fault tolerant topology control for wireless ad hoc networks," Wiley Journal on Wireless Communications and Mobile Computing, vol. 4, no. 1, pp. 109-125, 2004.

[20] M. Penrose, "The longest edge of the random minimal spanning tree," Annals of Applied Probability, vol. 7, pp. 340-361, 1997.

[21] C. Bettstetter, "On the minimum node degree and connectivity of a wireless multihop network," in ACM MobiHoc, 2002.

[22] C.-W. Yi, "Probabilistic aspects of wireless ad hoc networks," Ph.D. dissertation, Illinois Institute of Technology, 2005.

[23] P.-J. Wan, C.-W. Yi, "Asymptotic critical transmission radius for greedy forward routing in wireless ad hoc networks," in ACM MobiHoc, 2006.

[24] X.-Y. Li, P.-J. Wan, and Y. Wang, "Power efficient and sparse spanner for wireless ad hoc networks," in IEEE ICCCN, 2001, pp. 564-567.

[25] Qualnet network simulatior, http://www.scalable-networks.com/.

[26] D. B. Johnson and D. A. Maltz, "Dynamic source routing in ad hoc wireless networks," in Mobile Computing, Imielinski and Korth, Eds. Kluwer Academic Publishers, 1996, vol. 353. 